\title{
Hepatocellular carcinoma arising in adenoma: similar immunohistochemical and cytogenetic features in adenoma and hepatocellular carcinoma portions of the tumor
}

Sanjay Kakar ${ }^{1,2,5}$, James P Grenert ${ }^{1,5}$, Valerie Paradis ${ }^{3}$, Nicolas Pote ${ }^{3}$, Shriram Jakate ${ }^{4}$ and Linda D Ferrell ${ }^{1}$

${ }^{1}$ Department of Anatomic Pathology, University of California, San Francisco, CA, USA; ${ }^{2}$ Department of Pathology, Veteran Affairs Medical Center, San Francisco, CA, USA; ${ }^{3}$ Department of Pathology, Beaujon Hospital, Clichy, France and ${ }^{4}$ Department of Pathology, Rush University, Chicago, IL, USA

\begin{abstract}
Well-differentiated hepatocellular carcinoma in non-cirrhotic liver can show morphological features similar to hepatocellular adenoma. In rare instances, hepatocellular carcinoma can arise in the setting of hepatocellular adenoma. This study compares the immunohistochemical and cytogenetic features of the hepatocellular adenoma-like and hepatocellular carcinoma portions of these tumors. Immunohistochemistry for $\beta$-catenin, glutamine synthetase, serum amyloid A protein, glypican-3, and heat-shock protein 70 was done in 11 cases of hepatocellular carcinoma arising in hepatocellular adenoma in non-cirrhotic liver. Tumors with nuclear $\beta$-catenin and/or diffuse glutamine synthetase were considered $\beta$-catenin activated. Fluorescence in situ hybridization (FISH) was done in nine cases for gains of chromosomes 1, 8 and MYC. There were seven men (33-75 years) and four women (29-65 years). Focal atypical morphological features were seen in hepatocellular adenoma-like areas in $7(64 \%)$ cases. Hepatocellular adenoma-like areas showed features of inflammatory hepatocellular adenoma in $7(64 \%)$ cases; 4 of these were also serum amyloid A-positive in the hepatocellular carcinoma portion. $\beta$-Catenin activation, heat-shock protein $\mathbf{7 0}$ positivity, and chromosomal gains on FISH were seen in the hepatocellular adenoma portion in $55 \%, 40 \%$, and $56 \%$ of cases, and $73 \%, 60 \%$, and $78 \%$ of cases in the hepatocellular carcinoma portion, respectively. In conclusion, the hepatocellular adenoma-like portion of most cases of hepatocellular carcinoma arising in hepatocellular adenoma shows features typically seen in hepatocellular carcinoma such as focal morphological abnormalities, $\beta$-catenin activation, heat-shock protein 70 expression, and chromosomal gains. Hepatocellular adenoma-like areas in these tumors, especially in men and older women, may represent an extremely well-differentiated variant of hepatocellular carcinoma, whereas the morphologically recognizable hepatocellular carcinoma portion represents a relatively higher grade component of the tumor.
\end{abstract}

Modern Pathology (2014) 27, 1499-1509; doi:10.1038/modpathol.2014.50; published online 18 April 2014

Hepatocellular adenoma is benign neoplasm that occurs more frequently in women and is often associated with oral contraceptives. Other settings in which hepatocellular adenoma has been described include use of anabolic steroids, glycogen storage diseases, and hepatic iron overload. ${ }^{1}$ In the

Correspondence: Professor S Kakar, MD, Department of Anatomic Pathology, University of California, 4150 Clement Street, San Francisco, CA 94121, USA.

E-mail: sanjay.kakar@ucsf.edu

${ }^{5} \mathrm{SK}$ and JPG are equal first authors.

Received 27 September 2013; revised 24 February 2014; accepted 25 February 2014; published online 18 April 2014 last few years, the incidence of hepatocellular adenoma has increased, presumably related to non-alcoholic fatty liver disease. ${ }^{2}$ The 2010 WHO classification recognizes four subtypes of hepatocellular adenoma: hepatocyte nuclear factor 1 alpha $(H N F 1 \alpha)$-inactivated, $\beta$-catenin-activated, inflammatory, and unclassified. ${ }^{3-5}$

In rare instances, hepatocellular carcinoma can arise in the setting of hepatocellular adenoma. The reported risk in the literature ranges from 0 to $18 \%$; the figures based on the three large series are between 4 and $8 \% .1,2,6,7$ A recent study that extensively reviewed all reported cases in the literature estimated the risk to be $4.2 \%{ }^{8}$ In most instances, the 
hepatocellular carcinoma is diagnosed concurrently with the hepatocellular adenoma, whereas subsequent occurrence of hepatocellular carcinoma is less common. ${ }^{8}$ The $\beta$-catenin-activated adenomas are more likely to be associated with concurrent or subsequent diagnosis of hepatocellular carcinoma. ${ }^{4}$

Well-differentiated hepatocellular carcinoma in non-cirrhotic liver can show morphological features similar to hepatocellular adenoma, and can be indistinguishable from hepatocellular adenoma in some instances. Recurrence and metastasis have been reported in such well-differentiated tumors. ${ }^{9}$ The immunohistochemical and cytogenetic features of these well-differentiated neoplasms more closely resemble hepatocellular carcinoma than hepatocellular adenoma. ${ }^{9,10}$ In view of the close morphologic resemblance of hepatocellular adenoma and hepatocellular carcinoma, it is possible that the hepatocellular adenoma-like portion of tumors designated as 'hepatocellular carcinoma arising in adenoma' may represent an extremely well-differentiated form of hepatocellular carcinoma. In the WHO fascicle in 1999 Drs. Ishak and Goodman opined that the adenoma portion of these tumors is a 'grade 1' hepatocellular carcinoma. ${ }^{11}$ This study explores this possibility by comparison of immunohistochemical and cytogenetic features of the hepatocellular adenoma-like and hepatocellular carcinoma portions of tumors diagnosed as hepatocellular carcinoma arising in adenoma.

\section{Materials and methods}

\section{Study Population}

This study comprises 11 cases of hepatocellular carcinoma, which were apparently arising in hepatocellular adenoma in non-cirrhotic liver. Clinical information was recorded regarding oral contraceptive or steroid use and risk factors for fatty liver disease. All cases had both tumor components at the time of diagnosis. Morphological abnormalities like fatty change and atypical features (small cell change, thick cell plates, pseudoacinar architecture, cytologic atypia, reticulin loss) were noted for both components. By definition, hepatocellular adenoma component in none of the cases satisfied the International Working Party ${ }^{12,13}$ or World Health Organization criteria ${ }^{3}$ for the diagnosis for hepatocellular carcinoma and lacked features such as $>3$ cell thick plates, prominent cytologic atypia and loss of reticulin framework. None of the patients had chronic hepatitic or biliary disease, and there were no cases with bridging fibrosis or cirrhosis. Cases of hepatic adenomatosis, glycogen storage diseases, and Fanconi anemia were not included.

\section{Immunohistochemistry}

Immunohistochemistry was performed on formalinfixed paraffin-embedded tissue. The details of antibodies used for $\beta$-catenin, glutamine synthetase, serum amyloid A protein, glypican-3, and heatshock protein 70 are shown in Table 1.

\section{Scoring}

The staining intensity was graded 0-3 (absent, mild, moderate, strong). Glutamine synthetase was considered diffuse positive if moderate or strong staining was observed in $\geq 50 \%$ of tumor cells. Serum amyloid A and heat-shock protein 70 were scored positive if moderate or strong staining was seen in $\geq 10 \%$ of tumor cells. Glypican-3 was considered positive when moderate or strong nuclear/cytoplasmic/membranous staining was seen in $\geq 5 \%$ of tumor cells. Any nuclear staining with $\beta$-catenin was considered positive. Tumors with nuclear $\beta$-catenin and/or diffuse glutamine synthetase staining were considered $\beta$-catenin activated.

\section{Fluorescence In Situ Hybridization (FISH)}

FISH was done in nine cases for gains of chromosomes 1, 8 and c-myc. The Spectrum Orange-labeled probe against the centromeric region of chromosome 1 (CEP1), Spectrum Green-labeled probe against the centromeric region of chromosome 8 (CEP 8), and Spectrum Orange-labeled probe against c-myc were used (Abbott Molecular, Des Plaines, IL, USA). Fivemicrometer paraffin sections were baked at $55-60{ }^{\circ} \mathrm{C}$ for $30 \mathrm{~min}$ and deparaffinized, followed by DNA denaturation using $0.2 \mathrm{~N}$ hydrochloric acid for $20 \mathrm{~min}$, and pretreatment with $1 \mathrm{~mol} / \mathrm{l}$ of sodium thiocyanate for $30 \mathrm{~min}$ at $80^{\circ} \mathrm{C}$. The sections were then treated with protease $(0.5 \mathrm{mg} / \mathrm{ml}$ pepsin in $0.01 \mathrm{~N}$ hydrochloric acid) for $18 \mathrm{~min}$ at $37^{\circ} \mathrm{C}$. The probes were hybridized to the tissue overnight at $37^{\circ} \mathrm{C}$ in a moist chamber. The slides were washed in post-hybridization buffer $(2 \times$ standard saline citrate/

Table 1 Details of antibodies used for immunohistochemistry

\begin{tabular}{|c|c|c|c|}
\hline Antibody & Clone & Source & Dilution \\
\hline$\beta$-Catenin & 14 & BD Biosciences, San Jose, CA, USA & $1: 200$ \\
\hline Glutamine synthetase & Mab302 & Chemicon/Millipore, Billierica, MA, USA & $1: 250$ \\
\hline Serum amyloid A & mc1 & Dako, Carpinteria, CA, USA & $1: 50$ \\
\hline Glypican-3 & $1 \mathrm{G} 12$ & BioMosaics, Burlington, VT, USA & $5 \mu \mathrm{g} / \mathrm{ml}$ \\
\hline Heat-shock protein 70 & SC-24 & Santa Cruz Biotechnology, Santa Cruz, CA, USA & $1: 200$ \\
\hline
\end{tabular}


$0.3 \% \mathrm{NP}-40$ ) and counterstained with 2-6-diamidino-2-phenylindole. The signals were counted in at least 50 non-overlapping tumor nuclei per case, using the Zeiss Axio Imager fluorescence microscope (Carl Zeiss Imaging, Thornwood, NY, USA). The images were captured using Zeiss AxioVision imaging software.

Eleven counts of 50 cells each (total 550 cells) were performed for number of CEP1, CEP8, and $M Y C$ signals in normal hepatocyte nuclei, and mean and standard deviation were determined (Table 2). The mean number of cells per 100 cells with three or more signals was also determined for CEP1, CEP8, and $M Y C$. The normal upper limits were determined by using mean plus two standard deviations. Gains at CEP1, CEP8, and MYC loci were considered to be present if either of the following two conditions were met:

(1) The mean signal count per cell in the tumor exceeded the upper limit of normal (defined as mean signal count in normal cells plus 2 s.d.).

(2) Tumors with number of cells with three or more signals (per 100 cells counted) exceeded the upper limit of normal (defined as mean plus 2 s.d. of number of normal cells per 100 cells with three or more signals).

\section{Results}

\section{Clinical and Pathologic Characteristics}

There were seven men (33-75 years) and four women (30-65 years). Metabolic risk factors were present in $6(55 \%)$ patients (5 men, 1 woman; 2 obese, 2 diabetic, 2 both obese and diabetic). Two patients (both males) had history of anabolic steroids use. There were no discernible risk factors in the remaining three cases. The non-neoplastic liver showed steatosis or steatohepatitis in six cases, was normal in three cases and was not available for evaluation in two cases. Atypical morphological features were focally identified in hepatocellular adenoma-like areas in $7(64 \%)$ cases. These included small cell change (seven cases), pseudoacinar architecture (two cases), cytologic atypia (two cases), and focal loss of reticulin (three cases).

\section{Immunohistochemistry}

Inflammatory features. Serum amyloid A positivity was observed in hepatocellular adenoma-like areas in 7 (64\%) cases; 6 of these cases had typical histologic features of inflammatory hepatocellular adenoma including inflammation and prominent sinusoidal dilatation. Positive results with serum amyloid A were also observed in the hepatocellular carcinoma portion in four cases (Tables 3 and 4).

$\beta$-Catenin activation. Immunohistochemical evidence of activation of $\beta$-catenin was seen in hepatocellular adenoma portion in $6(55 \%)$ cases (Tables 3 and 4, Figures 1-3). Diffuse glutamine synthetase was seen in all six cases and was accompanied by nuclear $\beta$-catenin staining in four cases. $\beta$-Catenin activation in the hepatocellular adenoma portion was seen mostly in men $(71 \%$ vs $25 \%, P=0.1)$ and more often in patients older than 50 years $(67 \%$ vs $50 \%, P=0.4)$, but these associations were not statistically significant. Of the two women less than 50 years of age, $\beta$-catenin activation was not seen in the hepatocellular adenoma region.

In the hepatocellular carcinoma portion, 8 (73\%) cases had $\beta$-catenin activation (diffuse glutamine synthetase: 8 cases, nuclear $\beta$-catenin: 6 cases). For hepatocellular adenoma-like areas that were $\beta$-catenin activated and heat-shock protein 70 positive, $67 \%$ and $25 \%$, respectively, were in men (Tables 3 and 4).

Heat-shock protein 70 and glypican-3 staining. Positive staining with heat-shock protein 70 was observed in $4(40 \%)$ cases in hepatocellular adenoma portion and in $6(60 \%)$ cases in the hepatocellular carcinoma portion (Figure 3); results were not available for one case (Tables 3 and 4). None of the cases showed glypican-3 staining in hepatocellular adenoma or hepatocellular carcinoma portion.

\section{FISH}

FISH was performed on nine cases. CEP1, CEP8, and $M Y C$ signals were separately counted in hepatocel-

Table 2 FISH results in normal liver and in tumors

\begin{tabular}{lccc}
\hline & Mean & $\begin{array}{c}\text { Standard } \\
\text { deviation (s.d.) }\end{array}$ & $\begin{array}{c}\text { FISH criteria for } \\
\text { abnormal }\end{array}$ \\
\hline Mean CEP1 signals per cell +2 s.d.)
\end{tabular}

The mean normal counts were determined based on 11 counts of 50 cells each in normal hepatocyte nuclei. The upper limit of normal was established for average signal counts per tumor cell as well as number of cells with three or more signals per 100 tumor cells.

${ }^{a}$ All abnormal cases in the study had mean signal count of at least 2.4 (or higher), or $\geq 28$ cells per 100 tumor cells with three or more signal counts. 
Table 3 Clinical, immunohistochemical, and cytogenetic features of 11 cases of hepatocellular carcinoma arising in adenoma

\begin{tabular}{|c|c|c|c|c|c|c|c|c|}
\hline Age (years)/gender & $\begin{array}{l}\text { Non-neoplastic } \\
\text { liver }\end{array}$ & $\begin{array}{c}\text { Tumor } \\
\text { size }(\mathrm{cm})\end{array}$ & $\begin{array}{l}\text { Atypia in } \\
\text { HCA area }\end{array}$ & $\begin{array}{l}\beta \text {-Catenin } \\
\text { HCA HCC }\end{array}$ & $\begin{array}{l}\text { GS HCA } \\
\text { HCC }\end{array}$ & $\begin{array}{l}\text { SAA HCA } \\
H C C\end{array}$ & $\begin{array}{l}\text { HSP7O HCA } \\
\text { HCC }\end{array}$ & $\begin{array}{l}\text { FISH HCA } \\
\text { HCC }\end{array}$ \\
\hline $29 / F$ & NA & 30 & Absent & N P & N P & $\mathrm{P} \mathrm{N}$ & $\mathrm{N} \mathrm{N}$ & P P \\
\hline $75 / \mathrm{M}$ & Normal & 10 & Present & $\mathrm{N} \mathrm{N}$ & P P & $\mathrm{P} P$ & $\mathrm{~N} \mathrm{~N}$ & $\mathrm{~N} \mathrm{P}$ \\
\hline $63 / \mathrm{M}$ & Steatohepatitis & 7 & Absent & $\mathrm{N} \mathrm{N}$ & $\mathrm{N} \mathrm{N}$ & P P & $\mathrm{N} \mathrm{N}$ & P P \\
\hline $52 / \mathrm{M}$ & Normal & 19 & Present & N P & P P & $\mathrm{P} \mathrm{N}$ & N P & P P \\
\hline $60 / \mathrm{M}$ & Steatosis & 8 & Absent & $\mathrm{N} \mathrm{N}$ & $\mathrm{N} \mathrm{N}$ & $\mathrm{P} P$ & $\mathrm{~N} \mathrm{~N}$ & $\mathrm{~N} \mathrm{~N}$ \\
\hline $62 / \mathrm{M}$ & Steatohepatitis & 11 & Present & $\mathrm{P} P$ & P P & $\mathrm{N} \mathrm{N}$ & P P & P P \\
\hline $57 / \mathrm{F}$ & Steatosis & 15 & Present & $\mathrm{N} \mathrm{N}$ & $\mathrm{N} \mathrm{P}$ & $\mathrm{N} \mathrm{N}$ & P P & $\mathrm{N} \mathrm{P}$ \\
\hline $35 / \mathrm{M}$ & Steatosis & 7 & Present & $\mathrm{PP}$ & P P & $\mathrm{N} \mathrm{N}$ & ND ND & $\mathrm{P} \mathrm{P}$ \\
\hline $33 / \mathrm{M}$ & NA & NA & Present & P P & P P & P P & N P & $\mathrm{N} \mathrm{N}$ \\
\hline $65 / F$ & Normal & 30 & Present & P P & P P & $\mathrm{P} \mathrm{N}$ & P P & ND ND \\
\hline $30 / \mathrm{F}$ & NA & 8 & Absent & $\mathrm{N} \mathrm{N}$ & $\mathrm{N} \mathrm{N}$ & $\mathrm{N} \mathrm{N}$ & P P & ND ND \\
\hline
\end{tabular}

Abbreviations: NA, not available; ND, not done; $\beta$-catenin: $\mathrm{P}$, nuclear staining, $\mathrm{N}$, all other patterns; GS (glutamine synthetase): P, diffuse staining, $\mathrm{N}$, all other patterns; SAA (serum amyloid A) and HSP70 (heat-shock protein 70): P, positive, N, negative; fluorescence in situ hybridization (FISH): P, gains of chromosomes 1, 8, and/or MYC, N-all other results.

Table 4 Immunohistochemical and cytogenetic results in hepatocellular adenoma and hepatocellular carcinoma portions of the tumor

\begin{tabular}{lcc}
\hline & $\begin{array}{c}\text { Hepatocellular } \\
\text { adenoma area }\end{array}$ & $\begin{array}{c}\text { Hepatocellular } \\
\text { carcinoma area }\end{array}$ \\
\hline Nuclear $\beta$-catenin & $4(36)$ & $6(55)$ \\
Diffuse GS staining & $6(55)$ & $8(73)$ \\
SAA positive & $7(64)$ & $4(36)$ \\
GPC positive & 0 & 0 \\
HSP70 positive & $4(40)$ & $6(60)$ \\
Chromosomal $_{\text {abnormalities by FISH }}$ & $5(56)$ & $7(78)$ \\
\hline
\end{tabular}

Abbreviations: GS: glutamine synthetase; GPC: GLypican-3; HSP70: heat-shock protein; FISH: fluorescence in situ hybridization; SAA: serum amyloid associated protein.

Numbers in parenthesis reflect percentages.

${ }^{a}$ HSP70 staining information was available in 10 cases; FISH was done in 9 cases.

lular adenoma and hepatocellular carcinoma areas (Tables 2 and 3).

Hepatocellular adenoma area. Abnormal results were noted in $5(56 \%)$ cases (Figures 1, 2, and 4). Increased CEP1, CEP8, and MYC signals were seen in one case in the HCA area, whereas three cases had gain of CEP1 only and one case showed gain of $M Y C$ only. The chromosomal changes were seen mostly in males (four men and one woman), but there was no statistically significant association with age, gender, clinical risk factors, or histology of non-neoplastic liver. Of these 5 cases those who harbored cytogenetic abnormalities, $3(60 \%)$ showed $\beta$-catenin activation, but this association was not statistically significant.

Hepatocellular carcinoma area. Abnormal results were noted in $7(78 \%)$ cases. Increased CEP1, CEP8, and MYC signals were seen in six cases in the hepatocellular carcinoma area, whereas one case had gain of CEP1 only.

\section{Discussion}

Hepatocellular carcinoma arising in the setting of hepatocellular adenoma is a rare phenomenon and has been reported in $4-8 \%$ of cases., ${ }^{1,2,6-8}$ Both tumors occur concurrently in most reported cases, it has been assumed that this represents malignant transformation of an adenoma. ${ }^{14-20}$ Some reports have mentioned hepatocellular carcinoma arising in adenomas in the setting of underlying diseases like hepatitis B; it is likely that the adenoma part was a high-grade dysplastic nodule or well-differentiated hepatocellular carcinoma. ${ }^{21}$ Similarly, progression to hepatocellular carcinoma described in an adenoma where the latter diagnosis was based on a needle biopsy may have been an hepatocellular carcinoma at initial presentation that could not be diagnosed based on the biopsy specimen. ${ }^{22}$ In others, an adenoma with a subsequent recurrence as hepatocellular carcinoma at the same site has been described. ${ }^{23,24}$ It is quite likely that the original tumor in such cases was a well-differentiated hepatocellular carcinoma, which subsequently recurred. ${ }^{9}$ Most studies examining hepatocellular carcinoma arising in an adenoma were done before the new classification of HCA was adopted by the WHO in 2010. Farges et al have studied a large cohort of these patients using the current immunohistochemical markers in accordance with the World Health Organization classification. ${ }^{2}$ However, a detailed analysis of the hepatocellular adenoma and hepatocellular carcinoma portions of the tumor using a combination of immunohistochemistry (including heat-shock protein 70) and cytogenetic analysis has not been reported.

Advanced age, male gender, use of anabolic steroids, metabolic syndrome, and large tumor size have been cited as risk factors for hepatocellular carcinoma transformation in an adenoma. ${ }^{2,8}$ In our study, one or more of these risk factors were present in most cases: majority of the patients were males and older than 50 years, while metabolic risk factors 

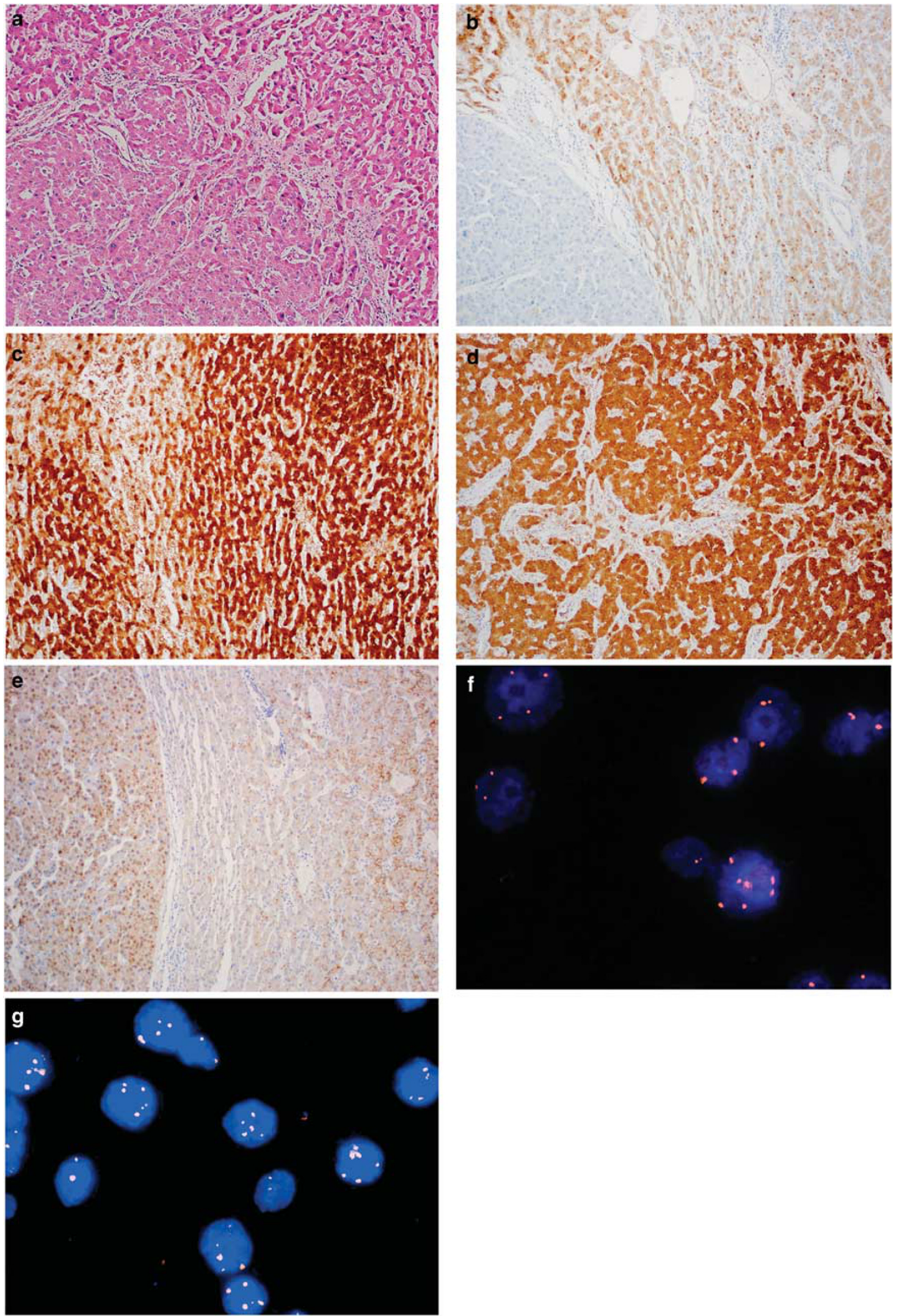

Figure 1 Adenoma-like (right) and hepatocellular carcinoma (left) portions in a 52-year-old man (a, H\&E, $\times 10)$. The adenoma-like portion showed inflammatory features and was positive for SAA $(\mathbf{b}, \times 10)$. Both adenoma-like $(\mathbf{c}, \times 10)$ and hepatocellular carcinoma portions $(\mathbf{d}$, $\times 20$ ) showed $\beta$-catenin activation as evidenced by diffuse glutamine synthetase staining; nuclear $\beta$-catenin was observed only in the HCC portion $(\mathbf{e}, \times 20)$. FISH showed chromosome 1 gains in both adenoma-like $(\mathbf{f}, \times 100)$ and hepatocellular carcinoma portions $(\mathrm{g}, \times 100)$. 

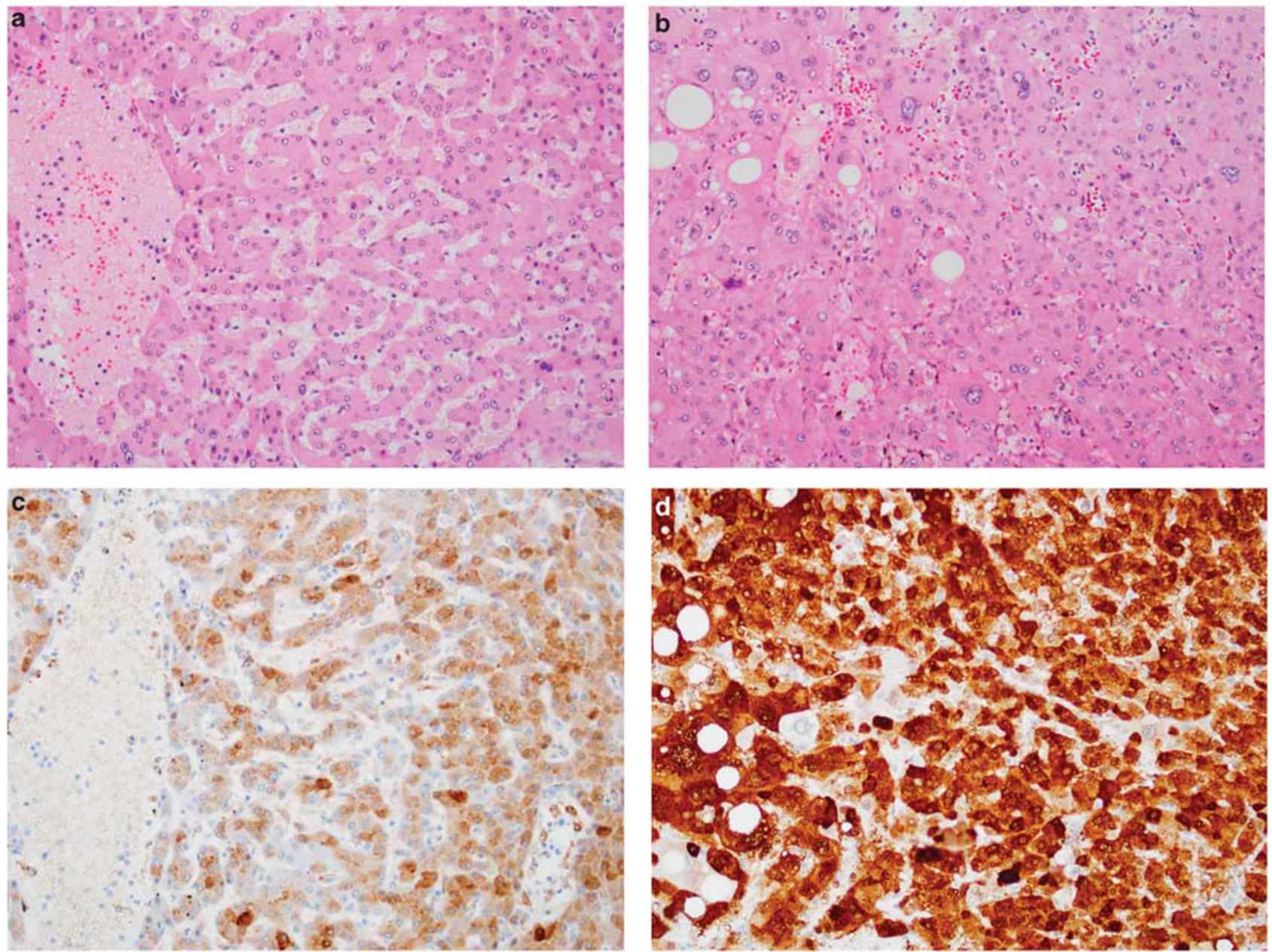

Figure 2 Adenoma-like $(\mathbf{a}, \mathrm{H} \& \mathrm{E}, \times 20)$ and hepatocellular carcinoma portion $(\mathbf{b}, \mathrm{H} \& \mathrm{E}, \times 20)$ in a tumor in a 33 -year-old male. There was $\beta$-catenin activation evidenced by diffuse glutamine synthetase staining in both adenoma-like $(\mathbf{c}, \times 20)$ and hepatocellular carcinoma portions $(\mathbf{d}, \times 20)$. The glutamine synthetase staining was stronger in the hepatocellular carcinoma portion. There were no chromosomal gains.

or use of anabolic steroids was present in nearly three-fourth of the cases. Based on the World Health Organization classification, the hepatocellular adenoma portion in two-thirds of cases showed features of inflammatory hepatocellular adenoma with or without $\beta$-catenin activation. Farges et al reported similar results with features of inflammatory hepatocellular adenoma in $56 \%$ of cases in the adenoma portion. ${ }^{2}$

In our study, activation of $\beta$-catenin was observed in the hepatocellular adenoma-like portion in $55 \%$ of cases, which is similar to $64 \%$ reported by Farges et $a .^{2}{ }^{2}$ As observed in earlier studies, $\beta$-catenin-activated tumors in our series occurred predominantly in men and showed atypical morphological features. Even though these tumors are currently classified as $\beta$-catenin-activated adenomas as per the 2010 World Health Organization classification, ${ }^{3}$ these are often associated with hepatocellular carcinoma at diagnosis or follow-up. ${ }^{4,7}$ As they often show cytogenetic changes similar to hepatocellular carcinoma, it has been proposed that these $\beta$-catenin-activated tumors represent extremely well-differentiated variants of hepatocellular carcinoma. ${ }^{10}$

Nuclear translocation of $\beta$-catenin leads to upregulation of glutamine synthetase, which manifests as strong and diffuse cytoplasmic expression. The concordance between nuclear $\beta$-catenin staining and diffuse glutamine synthetase staining is high, but some tumors show diffuse and strong glutamine synthetase expression in the absence of nuclear $\beta$-catenin staining. ${ }^{10} \mathrm{In}$ our study, this phenomenon was observed in HCA-like area in 33\% of cases, which is similar to the $29-44 \%$ range reported in other series. ${ }^{2,25,26}$ The reason for the discrepancy between glutamine synthetase and $\beta$-catenin expression is not known; some of these cases show $\beta$-catenin mutation without nuclear $\beta$-catenin or may have mutations affecting other components of the Wnt-signaling pathway like AXIN1 and AXIN2.4,27,28

Glypican-3 is an oncofetal antigen that is expressed in $70-90 \%$ of hepatocellular carcinomas, but 

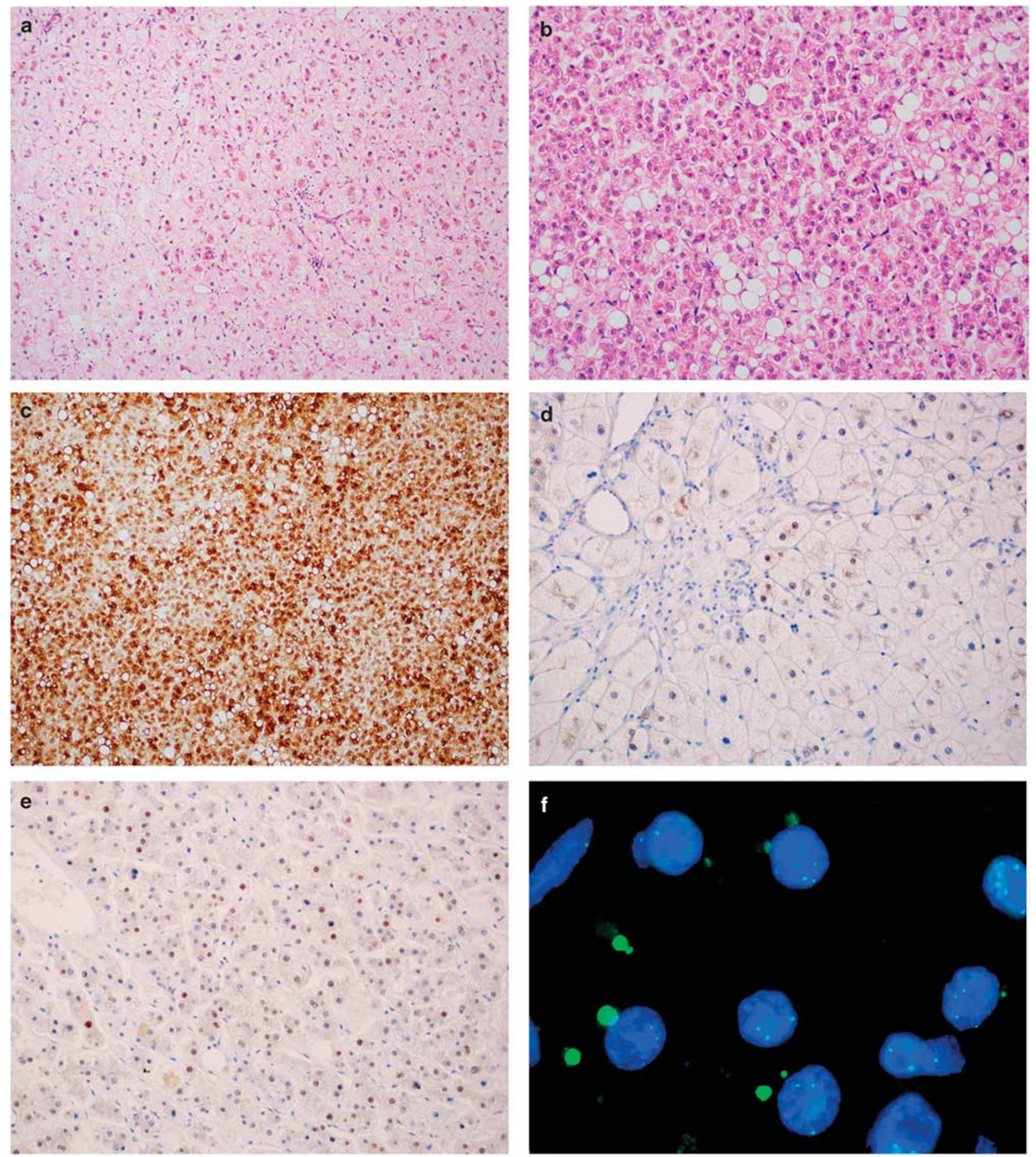

Figure 3 Adenoma-like $(\mathbf{a}, \mathrm{H} \& \mathrm{E}, \times 20)$ and hepatocellular carcinoma $(\mathbf{b}, \mathrm{H} \& \mathrm{E}, \times 20)$ portions of a tumor in a 61-year-old male. Both portions showed $\beta$-catenin activation evidenced by diffuse glutamine synthetase staining $(\mathbf{c}, \mathrm{HCC}, \times 10)$ and patchy nuclear $\beta$-catenin staining (d, adenoma-like, $\times 20)$. Patchy heat-shock protein 70 staining (e, adenoma-like, $\times 20)$ and chromosome 8 gain $(\mathbf{f}$, HCC, $\times 100)$ were observed in both portions.

not in normal adult liver and hepatocellular adenoma. ${ }^{29-31}$ We did not observe glypican-3 expression in the hepatocellular adenoma or hepatocellular carcinoma portion in any case. As the sensitivity of glypican-3 is low in well-differentiated hepatocellular carcinoma, ${ }^{31}$ these results are not surprising.

Heat-shock protein 70 is an anti-apoptotic protein and its overexpression allows cell survival. In a study comparing gene expression between 

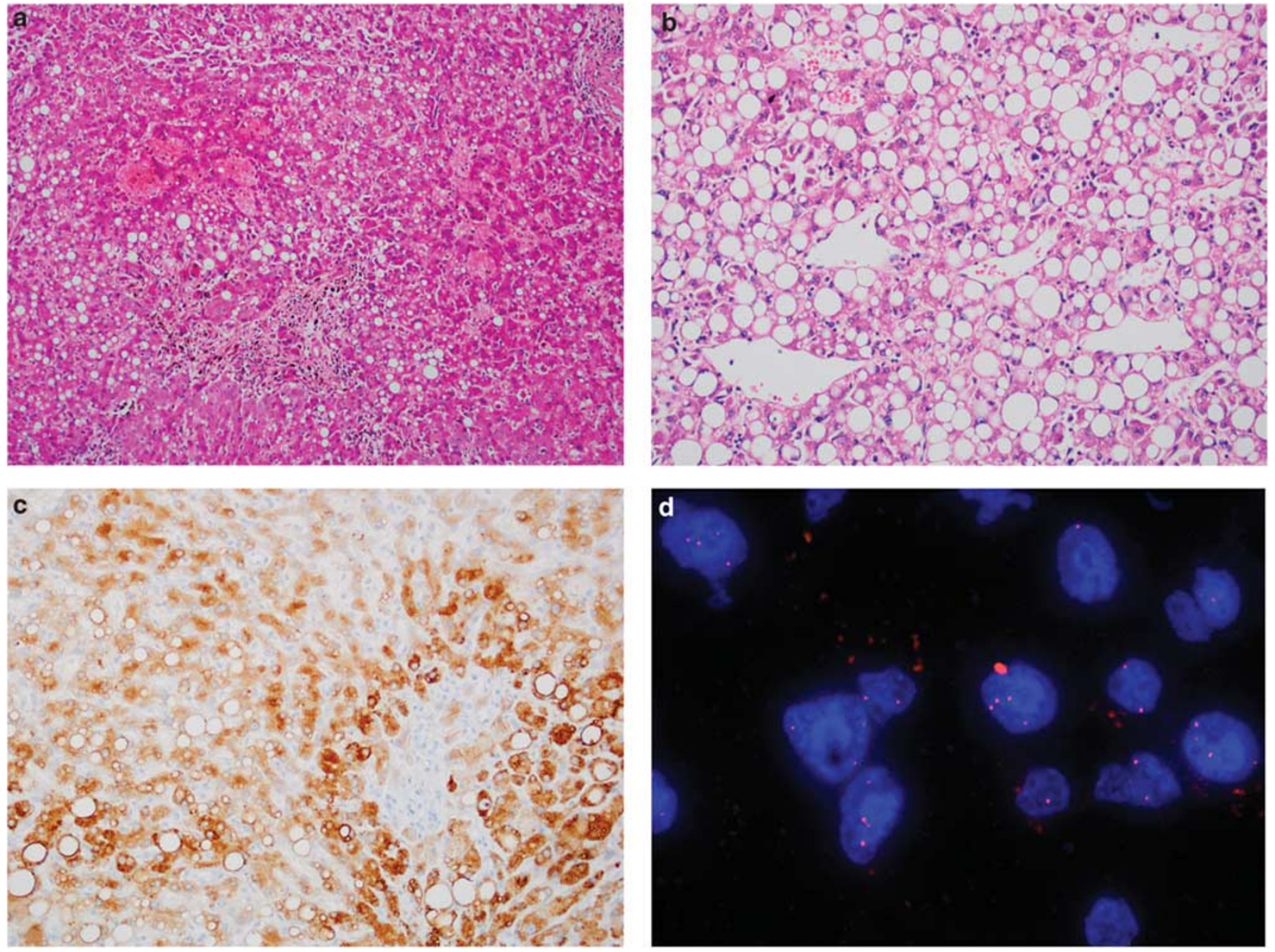

Figure 4 Adenoma-like $(\mathbf{a}, \mathrm{H} \& \mathrm{E}, \times 20)$ and HCC portions $(\mathbf{b}, \mathrm{H} \& \mathrm{E}, \times 20)$ of a tumor in a 63-year-old male. Both portions were serum amyloid A-positive $(\mathbf{c}$, adenoma-like, $\times 20)$ and showed chromosome 8 gain $(\mathbf{d}$, hepatocellular carcinoma, $\times 100)$. There was no $\beta$-catenin activation.

hepatocellular carcinoma and other hepatocellular nodules, heat-shock protein 70 was the most discriminatory gene. ${ }^{32}$ The utility of heat-shock protein 70 staining for the distinction of hepatocellular carcinoma from dysplastic nodules has been described. ${ }^{33,34}$ There is limited information about heat-shock protein 70 staining in hepatocellular adenoma, with numbers ranging from 0 to $13 \%$ in two studies. ${ }^{35,36}$ In our study, heat-shock protein 70 was expressed in the hepatocellular adenoma area in $40 \%$ of cases, further strengthening the argument that these areas may be extremely well-differentiated hepatocellular carcinoma.

Gains of part or the entire chromosome arm of $1 \mathrm{q}$ and $8 \mathrm{q}$ are the earliest and most common abnormalities in hepatocellular carcinoma. ${ }^{37-39}$ These abnormalities have not been reported in typical hepatocellular adenomas in young women, ${ }^{9,40}$ but can occur in adenoma-like tumors in men or older women. ${ }^{9}$ It has been proposed that these latter tumors represent well-differentiated hepatocellular carcinoma. In the present study, gains of chromosome 1 and/or 8 were observed in the hepatocellular adenoma-like area in 56\% of cases and in hepatocellular carcinoma portion in $78 \%$ of cases.

Our results show that atypical morphological features are often present in the HCA portion in tumors designated as hepatocellular carcinoma arising in hepatocellular adenoma. In the majority of cases, the hepatocellular adenoma-like portion also shows immunohistochemical ( $\beta$-catenin activation or heat-shock protein 70 positivity) and cytogenetic abnormalities (gains of chromosomes 1 and 8) similar to hepatocellular carcinoma. This provides support to the argument that the hepatocellular adenoma-like portion represents an extremely well-differentiated hepatocellular carcinoma in at least a subset of these cases. Although there is variability in the literature about the clinicopathologic associations of $\beta$-catenin activation in hepatocellular carcinoma, it has been correlated with older age, ${ }^{41}$ well-differentiated tumors, ${ }^{17,25,26}$ large size,${ }^{26}$ and a good prognosis. ${ }^{17,26,41,42}$ It is understandable that such well-differentiated tumors that have often grown to a large size without overt features of hepatocellular carcinoma or aggressive behavior 
have been labeled as an adenoma. However, recurrence and metastasis have been associated with these tumors. ${ }^{9}$ Case reports of hepatocellular carcinoma recurrences at sites of hepatocellular adenoma may also represent recurrence of welldifferentiated hepatocellular carcinomas that were diagnosed as hepatocellular adenoma. ${ }^{23,24}$ Experimental evidence in mouse models has shown that cooperation of Met and $\beta$-catenin activation leads to hepatocellular carcinoma, whereas cooperation of Met and defective signaling through the transcription factor HNF1 $\alpha$ leads to hepatocellular adenoma. $^{43}$ If the molecular mechanisms of hepatocellular adenoma and hepatocellular carcinoma pathogenesis are distinct as indicated by this experimental data, it appears less likely for hepatocellular adenoma to progress to hepatocellular carcinoma. It is more likely that in most such cases, the hepatocellular adenoma-like portion is an extremely well-differentiated hepatocellular carcinoma that has progressed to a morphologically overt hepatocellular carcinoma leading to an appearance of 'hepatocellular carcinoma arising in an adenoma'. A similar argument was recently presented by Witjes et al based on their review of hepatocellular carcinoma in non-cirrhotic liver, where a transition zone from hepatocellular adenoma to hepatocellular carcinoma was not identified in any case. ${ }^{44}$

A group of international liver pathologists has recently proposed that tumors with histologic features of adenoma with atypical features should be categorized as well-differentiated hepatocellular neoplasm with uncertain malignant potential (HUMP). ${ }^{45,46}$ As per this proposal, tumors resembling adenomas in men, and women $>50$ years, focal atypical morphologic features (insufficient for an unequivocal diagnosis of hepatocellular carcinoma), $\beta$-catenin activation and glypican-3 positivity should be classified as hepatocellular neoplasm with uncertain malignant potential. In addition, hepatocellular neoplasm with uncertain malignant potential should also be considered for tumors with strong heat-shock protein 70 staining, gains of chromosome 1 and 8 , and in unusual clinical settings such as glycogen storage disease and Fanconi anemia. Based on morphologic criteria alone, 64\% of adenoma-like areas in this study would be classified as hepatocellular neoplasm with uncertain malignant potential, but $91 \%$ of cases would have been classified as hepatocellular neoplasm with uncertain malignant potential based on a combination of clinical, morphologic, immunohistochemical, and cytogenetic features. In the study by Farges et al, the adenoma-like area in at least $80 \%$ of cases would have been classified as hepatocellular neoplasm with uncertain malignant potential. As detailed immunohistochemical results are not available in most reports of hepatocellular carcinoma in adenoma in the literature, it is difficult to estimate how many such tumors would have been classified as hepatocellular neoplasm with uncertain malignant potential. Although our results show that adenoma-like areas in cases with hepatocellular carcinoma in adenoma would fall under hepatocellular neoplasm with uncertain malignant potential category in an overwhelming number of cases, our data set is limited and do not rule out the possibility of hepatocellular carcinoma arising in conventional hepatocellular adenoma without atypical features.

In conclusion, the adenoma portion of tumors designated as hepatocellular carcinoma arising in adenoma often show focal morphological abnormalities, inflammatory features, $\beta$-catenin activation, heat-shock protein 70 expression, and gains of chromosomes 1 and/or 8. These findings indicate that the adenoma portion is likely to represent an extremely well-differentiated variant of hepatocellular carcinoma, especially in men and older women. The morphologically recognizable hepatocellular carcinoma portion is likely to represent a relatively higher grade component of the tumor.

\section{Disclosure/conflict of interest}

The authors declare no conflict of interest.

\section{References}

1 Dokmak S, Paradis V, Vilgrain V, et al. A single-center surgical experience of 122 patients with single and multiple hepatocellular adenomas. Gastroenterology 2009;137:1698-1705.

2 Farges O, Ferreira N, Dokmak S, et al. Changing trends in malignant transformation of hepatocellular adenoma. Gut 2011;60:85-89.

3 Biuolac-Sage P, Balabaud C, Wanless I. Focal nodular hyperplasia and hepatocellular adenoma. In: Bosman FT, Carneiro F, Hruban RH, Theise ND (eds). WHO Classification of Tumours of the Digestive System, 4th edn. IARC press: Lyon 2010; Chapter 10: pp 198-204.

4 Zucman-Rossi J, Jeannot E, Nhieu JT, et al. Genotypephenotype correlation in hepatocellular adenoma: new classification and relationship with HCC. Hepatology 2006;43:515-524.

5 Bioulac-Sage P, Rebouissou S, Thomas C, et al. Hepatocellular adenoma subtype classification using molecular markers and immunohistochemistry. Hepatology 2007;46:740-748.

6 Deneve JL, Pawlik TM, Cunningham S, et al. Liver cell adenoma: a multicenter analysis of risk factors for rupture and malignancy. Ann Surg Oncol 2009;16: 640-648.

7 Bioulac-Sage P, Laumonier H, Couchy G, et al. Hepatocellular adenoma management and phenotypic classification: the Bordeaux experience. Hepatology 2009; 50:481-489.

8 Stoot JH, Coelen RJ, De Jong MC, et al. Malignant transformation of hepatocellular adenomas into hepatocellular carcinomas: a systematic review including 
more than 1600 adenoma cases. HPB (Oxford) 2010;12: 509-522.

9 Kakar S, Chen X, Ho C, et al. Chromosomal abnormalities determined by comparative genomic hybridization are helpful in the diagnosis of atypical hepatocellular neoplasms. Histopathology 2009;55: 197-205.

10 Evason KJ, Grenert JP, Ferrell LD, et al. Atypical hepatocellular adenoma-like neoplasms with $\beta$-catenin activation show cytogenetic alterations similar to welldifferentiated hepatocellular carcinomas. Hum Pathol 2013;44:750-758.

11 Ishak KG, Goodman ZD, Stocker JT. Benign hepatocellular tumors. In: Tumors of the Liver and Intrahepatic Bile Ducts. AFIP press: Washington DC, 1999; Chapter 2 p21.

12 Ferrell LD, Crawford JM, Dhillon AP, et al. Proposal for standardized criteria for the diagnosis of benign, borderline, and malignant hepatocellular lesions arising in chronic advanced liver disease. Am J Surg Pathol 1993;17:1113-1123.

13 Anonymous, International Working Party. Terminology of nodular hepatocellular lesions. Hepatology 1995; 22:983-993.

14 Davis M, Portmann B, Searle M, et al. Histological evidence of carcinoma in a hepatic tumour associated with oral contraceptives. Br Med J 1975;4:496-498.

15 Korula J, Yellin A, Kanel G, et al. Hepatocellular carcinoma coexisting with hepatic adenoma. Incidental discovery after long-term oral contraceptive use. West J Med 1991;155:416-418.

16 Ferrell LD. Hepatocellular carcinoma arising in a focus of multilobular adenoma. A case report. Am J Surg Pathol 1993;17:525-529.

17 Chuang WY, Chen TC, Hsu HL, et al. Liver cell adenoma with concomitant hepatocellular carcinoma: report of two cases. J Formos Med Assoc 2002;101: 798-802.

18 Burri E, Steuerwald M, Cathomas G, et al. Hepatocellular carcinoma in a liver-cell adenoma within a noncirrhotic liver. Eur J Gastroenterol Hepatol 2006; 18:437-441.

19 Colovic R, Grubor N, Micev M, et al. Hepatocellular adenoma with malignant alteration. Hepatogastroenterology 2007;54:386-388.

$20 \mathrm{Kim} \mathrm{DH}$, Kim SU, Nam DH, et al. A case of hepatocellular carcinoma within hepatocellular adenoma in a non-cirrhotic male. Korean J Intern Med 2009;24:147-152.

21 Seo JM, Lee SJ, Kim SH, et al. Hepatocellular carcinoma arising from hepatocellular adenoma in a hepatitis B virus-associated cirrhotic liver. Clin Radiol 2012;67:329-333.

22 Gyorffy EJ, Bredfeldt JE, Black WC. Transformation of hepatic cell adenoma to hepatocellular carcinoma due to oral contraceptive use. Ann Intern Med 1989;110: 489-490.

23 Tesluk H, Lawrie J. Hepatocellular adenoma. Its transformation to carcinoma in a user of oral contraceptives. Arch Pathol Lab Med 1981;105:296-299.

24 Gordon SC, Reddy KR, Livingstone AS, et al. Resolution of a contraceptive-steroid-induced hepatic adenoma with subsequent evolution into hepatocellular carcinoma. Ann Intern Med 1986;105:547-549.

25 Audard V, Grimber G, Elie C, et al. Cholestasis is a marker for hepatocellular carcinomas displaying beta-catenin mutations. J Pathol 2007;212:345-352.
26 Dal Bello B, Rosa L, Campanini N, et al. Glutamine synthetase immunostaining correlates with pathologic features of hepatocellular carcinoma and better survival after radiofrequency thermal ablation. Clin Cancer Res 2010;16:2157-2166.

27 Taniguchi K, Roberts LR, Aderca IN, et al. Mutational spectrum of beta-catenin, AXIN1, and AXIN2 in hepatocellular carcinomas and hepatoblastomas Oncogene 2002;21:4863-4871.

28 Kim YD, Park CH, Kim HS, et al. Genetic alterations of Wnt signaling pathway-associated genes in hepatocellular carcinoma. J Gastroenterol Hepatol 2008;23: 110-118.

29 Yamauchi N, Watanabe A, Hishinuma M, et al. The glypican 3 oncofetal protein is a promising diagnostic marker for hepatocellular carcinoma. Mod Pathol 2005;18:1591-1598.

30 Wang XY, Degos F, Dubois S, et al. Glypican-3 expression in hepatocellular tumors: diagnostic value for preneoplastic lesions and hepatocellular carcinomas. Hum Pathol 2006;37:1435-1441.

31 Shafizadeh N, Ferrell LD, Kakar S. Utility and limitations of glypican-3 expression for the diagnosis of hepatocellular carcinoma at both ends of the differentiation spectrum. Mod Pathol 2008;21: 1011-1018.

32 Chuma M, Sakamoto M, Yamazaki K, et al. Expression profiling in multistage hepatocarcinogenesis: identification of HSP70 as a molecular marker of early hepatocellular carcinoma. Hepatology 2003;37: 198-207.

33 Di Tommaso L, Franchi G, Park YN, et al. Diagnostic value of HSP70, glypican 3, and glutamine synthetase in hepatocellular nodules in cirrhosis. Hepatology 2007;45:725-734.

34 Di Tommaso L, Destro A, Seok JY. Balladore et al. The application of markers (HSP70 GPC3 and GS) in liver biopsies is useful for detection of hepatocellular carcinoma. J Hepatol 2009;50:746-754.

35 Lagana SM, Salomao M, Bao F, et al. Utility of an immunohistochemical panel consisting of glypican-3, heat-shock protein-70, and glutamine synthetase in the distinction of low-grade hepatocellular carcinoma from hepatocellular adenoma. Appl Immunohistochem Mol Morphol 2013;21:170-176.

36 Nguyen T, Kakar S. Utility of HSP70, glutamine synthethase and glypican-3 in distinguishing hepatocellular adenoma from well-differentiated hepatocellular carcinoma. Mod Pathol 2012;25:420A (abstract 1753).

37 Balsara BR, Pei J, De Rienzo A, et al. Human hepatocellular carcinoma is characterized by a highly consistent pattern of genomic imbalances, including frequent loss of 16q23.1-24.1. Genes Chromosomes Cancer 2001;30:245-253.

38 Poon TC, Wong N, Lai PB, et al. A tumor progression model for hepatocellular carcinoma: bioinformatic analysis of genomic data. Gastroenterology 2006;131: 1262-1270.

39 Wilkens L, Bredt M, Flemming P, et al. Differentiation of liver cell adenomas from well-differentiated hepatocellular carcinomas by comparative genomic hybridization. J Pathol 2001;193:476-482.

40 Wilkens L, Bredt M, Flemming P, et al. Diagnostic impact of fluorescence in situ hybridization in the differentiation of hepatocellular adenoma and welldifferentiated hepatocellular carcinoma. J Mol Diagn 2001;3:68-73. 
41 Mao TL, Chu JS, Jeng YM, et al. Expression of mutant nuclear beta-catenin correlates with non-invasive hepatocellular carcinoma, absence of portal vein spread, and good prognosis. J Pathol 2001;193:95-101.

42 Fujito T, Sasaki Y, Iwao K, et al. Prognostic significance of beta-catenin nuclear expression in hepatocellular carcinoma. Hepatogastroenterology 2004;51:921-924.

43 Tward AD, Jones KD, Yant S, et al. Distinct pathways of genomic progression to benign and malignant tumors of the liver. Proc Natl Acad Sci USA 2007;104:14771-14776.
44 Witjes CD, Ten Kate FJ, van Aalten SM, et al. Hepatocellular adenoma as a risk factor for hepatocellular carcinoma in a non-cirrhotic liver: a plea against. Gut 2012;61:1645-1646.

45 Bedossa P, Burt AD, Brunt EM, et al. Well differentiated hepatocellular neoplasm of uncertain malignant potential (HUMP): Proposal for a new diagnostic category. Hum Pathol 2014;45:658-660.

46 Kakar S, Evason KJ, Ferrell LD. Well-differentiated hepatocellular neoplasms: getting over the 'HUMP'. Hum Pathol 2014;45:660-661. 